\section{Haemothorax caused by a solitary costal exostosis}

\author{
J R Reynolds, E Morgan
}

\begin{abstract}
A case of spontaneous haemothorax in a 14 year old boy due to trauma to the diaphragm caused by a solitary benign growth (exostosis) of a rib is described.
\end{abstract}

Exostoses are benign new growths protruding usually from the outer contour of a bone and characteristically capped by growing cartilage. Complications are rare, ${ }^{1-5}$ and only three cases of haemothorax have been reported. ${ }^{45} \mathrm{We}$ describe a case in which a single costal exostosis caused a sizeable haemothorax.

\section{Case report}

A 14 year old schoolboy was referred to hospital with a two week history of pain at the tip of his left shoulder aggravated by deep inspiration and movement. One day before admission to hospital he had a sudden onset of continuous pleuritic left sided chest pain associated with a feeling of light headedness and dyspnoea. There was no history of recent trauma.

He was a tall boy $(180 \mathrm{~cm})$, and on examination he was in obvious pain and respiratory distress. His pulse was 110 beats/min and the blood pressure was normal. There was dullness to percussion and reduced breath sounds at the left base. His haemoglobin concentration was $13.5 \mathrm{~g} / \mathrm{dl}$. Chest radiography showed a collection of fluid occupying half of the left hemithorax, and pleural aspiration disclosed pure blood (haemoglobin $13.0 \mathrm{~g} / \mathrm{dl}$ ). Cytological examination showed no abnormal cells and Gram stains and cultures showed no microorganisms. Clotting studies gave normal results.

He underwent a left thoracotomy, at which $1500 \mathrm{ml}$ of old blood was evacuated from the left pleural cavity. Initially no obvious source of the bleeding was evident but fresh clot was noted, adherent to the anterior aspect of the left hemidiaphragm adjacent to the insertion of the phrenic nerve. As he was exploring this area the surgeon had his finger pricked by a rose thorn like projection arising from the posterior aspect of the 7 th rib. This rib spur projected about $0.8 \mathrm{~cm}$ from the deep aspect of the rib, $2.5 \mathrm{~cm}$ posterior to the costochondral junction (figure). It was in direct contact with a small area of the diaphragm that was thickened and covered with friable tissue and adherent clot. There were no other abnormalities within the chest or rib cage.

The rib was resected and biopsy samples of the diaphragm were sent for histological examination. The rib section showed a benign exostosis with minimal cartilage remaining. The biopsy samples of the diaphragm showed vascular cellular fibrous tissue with a chronic inflammatory infiltrate. The patient made an uneventful and full recovery.

\section{Discussion}

Exostoses may occur singly as apparent isolated defects or multiply as a congenital disorder (hereditary multiple cartilaginous exostoses). In the multifocal hereditary disorder the lesions occur in infants and children and usually cease to enlarge by puberty. Isolated lesions also occur in infants, but are occasionally found in adults. At least $80 \%$ are discovered before the age of 21 . Isolated exostosis is found most often on the lower metaphysis of the femur and upper metaphysis of the tibia.

Complications arising from cartilaginous or bony exostoses are uncommon; but central and peripheral nerve compression. ${ }^{1}$ popliteal aneurysm, ${ }^{2}$ and traumatic haemarthrosis ${ }^{3}$ as well as haemothora ${ }^{45}$ have been reported. All three patients with haemothorax, however, had multiple cartilaginous exostoses unlike our patient, whose exostosis appeared to be truly solitary.

In our case (as in the previously reported cases) the sharp edge of the exostosis is likely to have caused direct trauma to the underlying viscera. In the previous cases trauma to the lung and pleura led to relatively minor



Radiograph showing the bony spur projecting from the inner aspect of the resected rib (the spur was fractured during surgery). 
haemothoraces, whereas our patient clearly displayed trauma to the surface of the diaphragm and a sizeable haemothorax. Close proximity of the phrenic nerve to the injured area probably explains our patient's shoulder tip pain, which was present for two weeks before his admission. The pain was not present after operation.
1 Madigan R, Worrall T, McClain EJ. Cervical cord compression in hereditary multiple exostosis. Review of the literature and report of a case. J Bone Joint Surg 1974; 56:401-4.

2 Holvelius L. Aneurysm of popliteal artery caused by cartilaginous exostosis. Orthop Scand 1975;46:836-8.

3 Hanrahan PS, Edelman J, Brash S. Spontaneous hemarthrosis associated with an exostosis of the talus. $J$ Rheumatol 1987;14:171.

4 Propper RA, Young LW, Wood BP. Hemothorax as a complication of costal cartilaginous exostosis. Pediatr Radiol 1980;9:135-7.

5 Balatskii AV, Rusinowich IuI, Reshetnikova IuK. Case of traumatic hemothorax in a child with multiple cartilaginous exostoses. Pediatriia (Rus) 1973;52:82-4. 